\title{
Software Support for Skeleton Recognition and Monitoring People with Privacy
}

\author{
Yoshihisa Nitta \\ Department of Computer Science \\ Faculty of Liberal Arts \\ Tsuda University \\ nitta@tsuda.ac.jp
}

\begin{abstract}
In this research, we have developed an open source software tool which makes it easy for a user to get skeleton information of people in a live image by use of Kinect for Windows V2. Our tool is a set of library software which provides users with easy coding to get body information and face recognition. The tool has been distributed widely and used by several users already for their research work such as robotics. In this paper, we propose possible use cases such as a remote monitor system for elderly care with privacy as well as a monitor system for shelters at disaster.
\end{abstract}

\section{Introduction}

It is popular for one to obtain skeleton information out of the images and moving pictures in motion captures.

The traditional way for skeleton recognition has been conducted by use of marker based systems [1][2][3]. A subject needs to put on markers around his/her body and the locations of markers are tracked and detected out of images and motion pictures taken by camera. Tracking would be done various ways such as optical motion picture and the use of an infrared camera. While marker-based systems have been used extensively, they are expensive.

Recently a more economical markerless systems, Kinect for Windows V2 [4] (hereinafter called "Kinect V2") is available. Woolford [5] suggests that such a system, the Kinect V2, it may be useable compare to a traditional clinical system, in a healthcare environment because the target person does not need to put on any marker device so that tracking would be done without making physical contact with that person. The Kinect V2 developed by Microsoft is a device with many functions such as skeleton tracking, face tracking and voice direction acquisition. Human body data can be obtained with sufficient accuracy without contact with a special marker on the human body.

\author{
Yuko Murayama \\ Department of Computer Science \\ Faculty of Liberal Arts \\ Tsuda University \\ murayama@tsuda.ac.jp
}

Leap motion devices are markerless as well. Unfortunately they are supposed to be used only for hands [6].

The original code API for $\mathrm{C}++$ with the Kinect for Windows SDK 2.0 consists of too many methods; 54 kinds of Interface, and the total number of methods is 277 [7]. We had opportunities for our university students to program Kinect V2 using the SDK, but they faced difficulties and could not be completed easily. The cause of difficulty was primarily due to the dependent function calls and troublesome memory management. So we felt the need to propose a slightly larger, more appropriate granularity API such that the number of methods would be reduced.

In this research, we have developed a class library NtKinect [8] [9] for C++ so that it is easier for programming to use the Kinect V2. The library has been released as an Open Source of MIT license.

With the library, one can easily perform skeleton tracking (Body Framework information) as well as face recognition [10][11]. The tool has been distributed widely and used by several users already for their research work such as robotics and computer art systems [12][13][14].

As an application of this library, we propose a watching over service is promising. There are various watching systems as classified in [15] . For watching services for the elderly, there are services that do not use IT technology, for example, post officers or electric and water inspectors visit homes at regular intervals to check the resident's health status [16]. However, in order to respond quickly to emergency situation, it is preferable to use IT technology. The Ramrock system [17] detects loitering and fall of an elderly alone for automatic warning, but sendnig images of a surveillance camera may cause privacy problems. In consideration of privacy, there are some methods using sensors other than camera to watch more loosely. In the system of Zojirushi [18], the usage records of a pot is sent twice a day, but such information is too indirect to detect emergency state. The system that watches the elderly 
by attaching sensors to furnitures such as a bed [19] has been studied, but it is inevitably a relatively large system. In the system of Philips [20] where a watched person wears a pendant type sensor for fall detection, there is a troublesome disadvantage that the sensor must be worn at all times. In the system of Fujitsu [21] which uses "sound analysis" of daily sound such as coughing and snoring to confirm the safety and estimates the health condition of elderly, the privacy is kept, but the accuracy of grasping the state of the elderly from the sound might become a problem.

From the above consideration, the method of recognizing the skeleton with non-attached sensor and directly grasping the human motion and posture is a superior method to watch people with privacy, and has a great advantage for other methods.

Furthermore, the recognized skeleton data has a very small size compared to camera images, so it is practical even if the data is sent over a narrow bandwidth network. This means a major advantage in terms of communication cost, especially when monitoring a large number of places, detacting a person's appearance and notifying it. This can be used for management of a place to be a shelter in case of a disaster.

This paper reports our work on the library software as and proposes some possible use cases of the Kinect V2 such as a remote monitor system for elderly care with privacy as well as a monitor system for shelters at disaster. The next section introduces the design and implementation of our library software. Section 3 shows an example of skeleton recognition program to express the granularity of our library's API. Section 4 shows the configuration of a system that distributes skeleton and face recognition results to a network as a server. Section 5 proposes two applications of the library for the remote monitor system. Section 6 gives some conclusions.

\section{The design of the library for Kinect V2}

In order to make best possible prospect of programming development using the function of Kinect $\mathrm{V} 2$, we have newly developed $\mathrm{C}++$ class library NtKinect.

The design policies for our library, NtKinect, are as follows:

- we use the "Kinect for Windows SDK 2.0" as the development base

- we take the object-oriented approach to manage devices

- we make use of the Standard Template Library (hereinafter called "STL") to manage the collection-class data
- we use the Open Source Computer Vision (OpenCV) data structure

- we use mutex and our library supports multi-threading

- it should be easy to make Dynamic Link Library (DLL)

In our library, all the sensors of a Kinect V2 are managed with one object. Data from sensors will be acquired by one method invocation and saved in the object. Each sensor is initialized at the first method call to get the data.

The number of data for body information detected by Kinect V2 changes from moment to moment, so that the data would be managed best as "Collection" data by the STL.

The STL is a library for $\mathrm{C}++$ programming language and provides some facilities including containers. We deal with data from Kinect V2 sensors with Collection, such as pair, vector and array of STL's sequence containers, not with simple array. In the Collection, the size may be changed at any time and each element is assigned on the heap memory.

OpenCV is useful for image processing and image recognition [22]. It becomes even easier to use in $\mathrm{C}++$ from version 2 or later. If one managed the data obtained from Kinect V2 with OpenCV data structure, the data could be passed directly to the OpenCV functions.

The OpenCV data structure, $c v::$ Mat, holds image data of Kinect V2, such as color, depth (distance), bodyindex, and infrared. Since each sensor uses a different coordinate system, we need to provide the functions for coordinate transformation (See Fig. 1 and Table. 1).

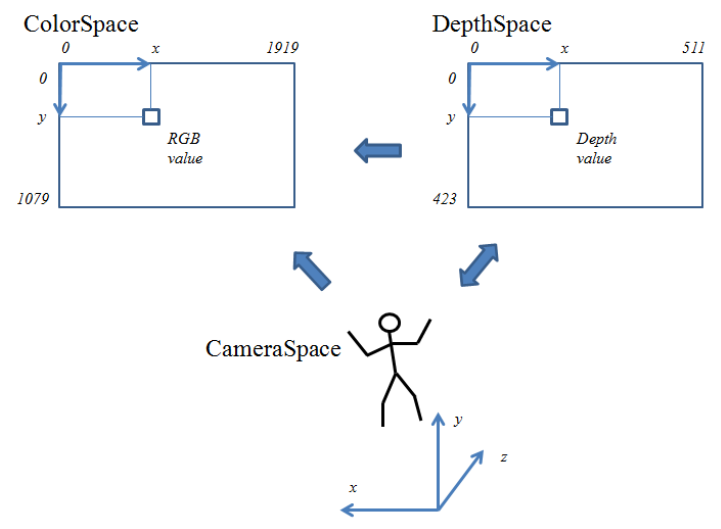

Figure 1. Data Representation of cv::Mat and Coordinates Transformation

Parallel processing may well be required for the following operations: 
Table 1. Data and Coordinates System of Kinect V2

\begin{tabular}{l|l}
\hline \hline Coordinates System & Types of Data \\
\hline ColorSpace & Color Image \\
DepthSpace & Depth Image, BodyIndex Image, Infrared Image \\
CameraSpace & Skeleton Information \\
\hline
\end{tabular}

- data acquisition from sensor devices

- the processing of acquired data

We provide a method that performs mutual exclusion control using "mutex" as well as thread-safe methods, so that the above two operations could be processed in a parallel way.

Due to our design policies, the NtKinect Object and the acquired data from sensors are allocated on the heap memory. Accordingly it becomes possible to make the developed program into DLLs so that it would be easier to use those programs in a different language and development environment.

We implemented a library based on the design policy described above. Method API is shown in Table 2 and member variables are shown in Table 3 . In those tables, arguments of methods and the types of some member variables are omitted.

Presumably the program repeats the following operation: data is stored in a variable in the object by one method call. Data would be accessed through variables from outside the object.

The current version of our library supports Kinect V2 skeleton recognition, face recognition, detailed face recognition (HDFace), gesture recognition, acquisition of sound and its direction, and speech recognition.

\section{Development of a program with NtKinect}

In this section, we show how one can use our library to write a program in $\mathrm{C}++$ for skeleton recognition. A sample program is shown in Fig. 2, and its execution result is Fig. 3. The program of Fig. 2 recognizes the skeleton and draws the joint position at the corresponding location on the camera image. For skeleton recognition, 25 joints positions per person are obtained in 3-dimensional coordinates, and their positions are superimposed and displayed on the 2-dimensional color image take by a camera. As can be seen from Fig. 2, the programs using Kinect V2 can be described more concisely than the traditional ones [23]. Therefore, the ovarall system is easy to understand for everyone, and effective reuse becomes possible. Of course, it can also be used to build a watching system.

The operation of the program is as follows.
- Call the kinect.setRGB() method to get the color 2-dimensional image taken by a camera.

- Call the kinect.setSkeleton() method to recognize skeleton.

- Since the positions of the joints are expressed in the CameraSpace coordinate system, they are converted to the ColorSpace coordinate system and then rectangles are drawn on the color image.

- Joint data has meaning only when its TrackingState member variable has the value other than TrackingState_NotTracked.

- Display the camera image in which the joints are drawn in red.

\section{Skeleton Data Server}

There is a need for a system that automatically recognizes the existence and state of people and communicates the result to a remote place. For this needs, skeleton recognition is considered to be a very useful means. The reasons are as follows.

- A system that watches people remotely is necessary, but since it is human beings being watched, privacy problems tend to occur. Abstracting human image data into skeleton information will protect their privacy.

- It is a laborious task in manpower to pick out only the images of people from many images. If it is possible to automatically select only the image of people and send it together with the information of the time and place, it is possible to save labor for monitoring.

We set up a system that sends out skeleton recognition result over the Internet using the NtKinect library. This server system is composed of a Note PC (Windows 10) with the Internet connection and Kinect V2 connected. The model of the system is shown in Fig. 4.

The server system keeps getting human skeleton information and voice within the distance between $50 \mathrm{~cm}$ and $450 \mathrm{~cm}$ from the front of Kinect V2. The acquired data will be sent in response to the 
Table 2. Methods of NtKinect.

\begin{tabular}{|c|c|c|c|}
\hline Category & Type & Method Name & Details (variable to be changed) \\
\hline RGB & void & setRGB & set Color Image to rgbImage \\
\hline Depth & void & setDepth & set Depth Image to depthImage \\
\hline BodyIndex & void & setBodyIndex & get Body Index Image to bodyIndexImage \\
\hline Infrared & void & setInfrared & set Infrared Image to infraredImage \\
\hline Skelton & void & setSkeleton & recognize Skeleton and set to skeleton, skeletonId, skeletonTrackingId \\
\hline Palm & pair $<$ int,int $>$ & handState & Recognize Hand State \\
\hline Face & void & setFace & Recognize Face and set to facePoint, faceRect, faceDirection, faceProperty \\
\hline Face & void & setHDFace & $\begin{array}{l}\text { Recognize HDFace and set to hdfaceVertices, hdfaceTrackingId, } \\
\text { hdfaceStatus }\end{array}$ \\
\hline Audio & void & setAudio & set Audio data to beamAngle, beamAngleConfidence, audioTrackingId \\
\hline Audio & void & drawAudioDirection & draw audio beam direction \\
\hline Audio & bool & isOpendAudio & flag for recording or not-recording \\
\hline Audio & void & opendAudio & start recording \\
\hline Audio & void & closeAudio & end recording \\
\hline Speech & void & setSpeechLang & Set language and words for recognition \\
\hline Speech & void & startSpeech & Start speech recognition \\
\hline Speech & void & stopSpeech & Stop speech recognition \\
\hline Speech & bool & setSpeech & Recognize Speech and set to recognizedSpeech, speechTag $\mid$ Item $\mid$ Confidence \\
\hline Gesture & void & setGestureFile & register Gesture difinition file \\
\hline Gesture & void & setGesture & $\begin{array}{l}\text { Recognize Gesture and set to discreteGesture, discreteGestureTrackingId, } \\
\text { continuousGesture, continuousGestureTrackingId }\end{array}$ \\
\hline
\end{tabular}

Table 3. Member variables of NtKinect.

\begin{tabular}{|c|c|c|c|}
\hline Category & Type & Name & Details \\
\hline RGB & cv::Mat & rgbImage & Color Image \\
\hline Depth & cv::Mat & depthImage & Depth Image \\
\hline BodyIndex & cv::Mat & bodyIndexImage & BodyIndex Image \\
\hline Infrared & cv::Mat & infraredImage & Infrared Image \\
\hline Skeleton & vector $<$ vector $<$ Joint $>>$ & skeleton & Skeleton Information \\
\hline Skeleton & vector $<$ int $>$ & skeletonId & Skeleton's BodyIndex \\
\hline Skeleton & vector $<$ INT64 $>$ & skeletonTrackingId & Skeleton's TrackingID \\
\hline Face & vector $<$ vector $<$ PointF $>>$ & facePoint & Position of Face Parts \\
\hline Face & vector $<\mathrm{cv}:$ Rect $>$ & faceRect & Boundingbox of Face \\
\hline Face & vector $<\mathrm{cv}:$ :Vec $3 \mathrm{f}>$ & faceDirection & Face Direction \\
\hline Face & vector $<$ vector $<$ DetectionResult $>>$ & faceProperty & Face Properties \\
\hline Face & vector $<$ UINT64 $>$ & faceTrackingID & Face's skeletonTrackingID \\
\hline HDFace & vector $<$ vector $<$ CameraSpacePoint $>>$ & hdfaceVertices & Position of HDFace Parts \\
\hline HDFace & vector $<$ UINT64 $>$ & hdfaceTrackingId & HDFace's skeletonTrackingId \\
\hline HDFace & vector $<$ pair $<$ int,int $>>$ & hdfaceStatus & HDFace properties \\
\hline Audio & float & beamAngle & Audio Beam's Direction \\
\hline Audio & float & beamAngleConfidence & beamangle Confidence \\
\hline Audio & UINT64 & audioTrackingId & Speaker's skeletonTrackingId \\
\hline Speech & bool & recognizedSpeech & Flag for Speech Recognition \\
\hline Speech & wstring & speechTag & Tag of Recognized Word \\
\hline Speech & wstring & speechItem & Item of Recognized Word \\
\hline Speech & float & speechConfidence & Confidence \\
\hline Speech & float & confidenceThreshold & Threshold \\
\hline Gesture & vector $<$ pair $<\ldots$,float $>>$ & discreteGesture & Discrete Gesture and it's confidence \\
\hline Gesture & vector $<$ UINT64 $>$ & discreteGestureTrackingId & Discrete Gesture's skeletonTrackingId \\
\hline Gesture & vector $<$ pair $<\ldots$.,float $>>$ & continuousGesture & Continuous Gesture and it's progress \\
\hline Gesture & vector $<$ UINT64 $>$ & continuousGestureTrackingId & Continuous Gesture's skeletonTrackingId \\
\hline
\end{tabular}




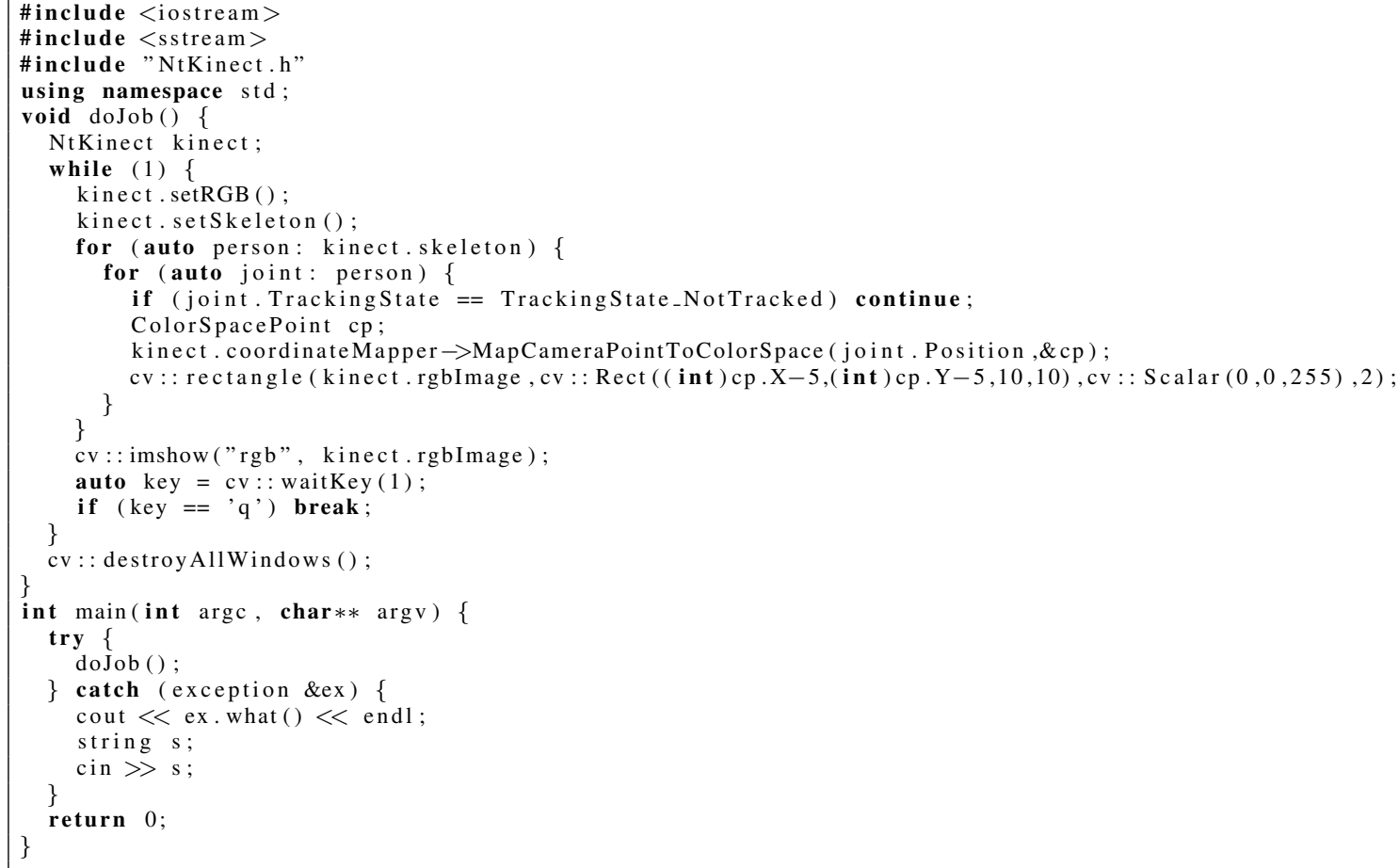

Figure 2. Recognition of Skeleton with NtKinect $\left(\mathrm{C}_{+}+\right)$. Kinect's Programs can be described more compactly than the traditional ones.

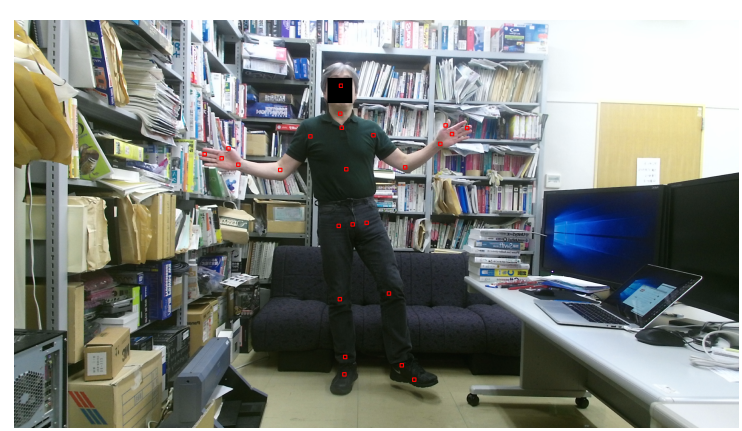

Figure 3. Execution Result of the Program in Fig. 2

requests from the client. The data includes as skeleton information as well as the information such as which skeleton has sounded or made a specific movement most recently. The client is authenticated with its IP Address or a password.

\section{Proposed Applications of Kinect V2}

In this section, we present two possible applications which make use of Kinect V2 so that our library would be useful. One is a remote monitor system for elderly care with privacy and the other is a monitor system for shelters at disaster to keep track automatically of the people. We describe our proposal in the following

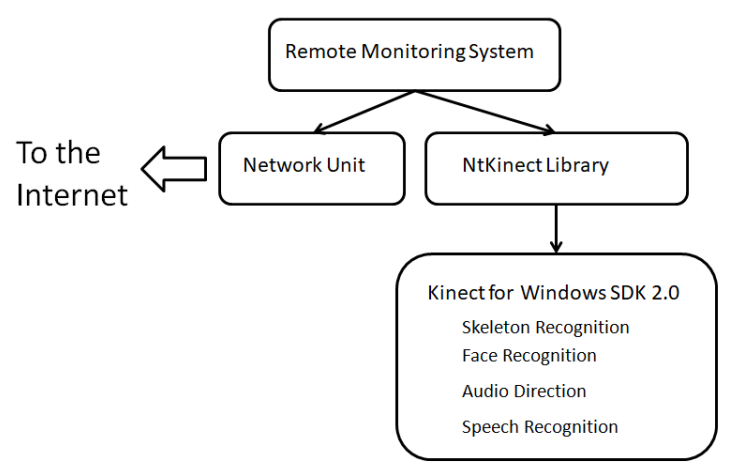

Figure 4. Remote Monitoring System

subsections.

\subsection{A remote monitor system for elderly care}

We have been facing a demographic problem of increasing population of elderly in Japan. A remote monitoring for their safety and security has been implemented to monitor those who live alone. As mentioned in Section 1, several attempts have been made with IT technology [17] [18] [19] [20] [21]. While these attempts are useful, there remain privacy issues and accurate grasp of people's state to be solved.

To find accidents and abnormalities by recognizing 
the skeleton, we could monitor the elderly for their safety and security with care for their privacy. Indeed, monitoring with the posture and gesture by skeleton recognition together with speaking sound and audio direction, we could watch over the safty and security from remotely as well. The outline of the system is shown in Fig. 5.

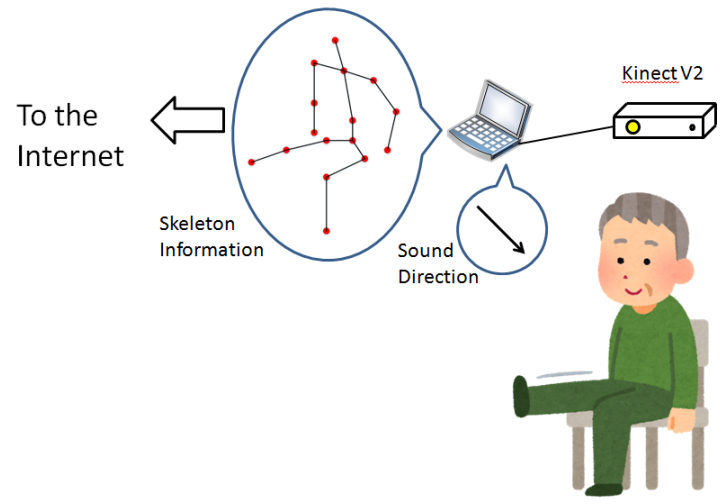

Figure 5. Watching the elderly with Privacy

\subsection{Detecting the status of shelters at disaster}

We have had various natural disasters almost every month in Japan[24]. One of them is the sudden volcano explosion with Mt. Ontake in Japan on September 27, 2014, causing a catastrophe in which 58 people were killed and 5 were still missing[25].

A lot of people evacuated to nearby mountain lodges in the event of the disaster, but the administrative side took time to grasp which evacuees are in which mountain lodges.

We suggested the needs for information processing at disaster by taking examples of the Great East Japan Earthquake at March 11, 2011 [26].

At large-scale disasters, it is highly crucial to grasp the information urgently on who or how many people are evacuated in what shelters. If such information is collected manually, there would be a delay in rescue and emergency response. If it is possible to detect the possible existence of people automatically, there will be a high possibility that the rescue and response will be improved dramatically [27].

Moreover, public places such as community centers and halls are likely to be utilized as evacuation centers at disaster. If we monitor the people around in those buildings not only at disaster but also at normal situations, there might be a privacy issue coming up that people feel that they are monitored by identified who they are. On the other hand, with our system with "skeleton information," we can detect the existence of people without collecting personal information so that privacy may well be preserved presumably.

Another issue is the amount of data to be transferred over the network for communication with our proposed monitoring method with Kinect V2 compared to the use of traditional monitor with live streaming or images. Since one joint position is represented by three float numbers, when expressing skeleton information of a human with 25 joints, it can be represented by 4 bytes $\times 3 \times 25=300$ bytes. We presume it absolutely useful in case of disaster with limited bandwidth available for communications [28].

\section{Conclusion}

In this paper, we report the development a libray software, NtKinect, which makes it easier to use a Kinect V2 device and the possible applications to use such a device so that the library software would be used to a great extent.

The library has been released as an Open Source of MIT license since July 2016 and used widely for various applications such as in collaborative robotics and computer art systems. With our library, it is possible to automatically recognize the existence of human beings and his/her postures and gestures, so the system could be used for monitoring the elderly people as well as detecting victims for situation awareness at a disaster.

Considering the generalization of low-speed mobile communication for IoT in the near future, it seems that utilizing the result of skeleton recognition becomes increasingly important. With recent mobile communication systems for Internet of Things (IoT), it is possible to use a network which is inexpensive but slower. LPWAN (Low-Power Wide-Are Network) [29] is such a wireless telecommunication wide area network to provide long range communications at a low bit rate among connected objects. For example, SIGFOX [30] data rates is $100 \mathrm{bps}$, and LoRaWAN [31] data rates range from $0.3 \mathrm{kbps}$ to $50 \mathrm{kbps}$. With the $5 \mathrm{G}$ mobile communication system (the fifth generation mobile networks), which is coming into use widely, since IoT communication becomes popular in many applications, it will be certainly necessary to communicate with as small traffic volume as possible. According to such trend, our proposed use of skeleton information to detect the existence of people could be useful.

\section{References}

[1] Sementille,A.C., Loureno, L.E., Brega,J.R.F. and Rodello, I.: A motion capture system using passive markers, Proc. of the 2004 ACM SIGGRAPH international conference on 
Virtual Reality continuum and its applications in industry (VRCAI '04), pp.440-447, 2004

[2] Barber, A., Cosker, D., James , O.,Waine, T. and Patel, R.: Camera tracking in visual effects an industry perspective of structure from motion, ACM Proc. of the 2016 Symposium on Digital Production (DigiPro '16) pp.45-54, 2016.

[3] Schrder, M., Maycock, J. and Botsch, M.: Reduced marker layouts for optical motion capture of hands, Proc of the 8th ACM SIGGRAPH Conference on Motion in Games (MIG '15), pp.7-16, 2015.

[4] Microsoft: Meet Kinect for Windows, https://developer.microsoft.com/en-us/windows/kinect (2017/06/08 access).

[5] Woolford, K.: Defining accuracy in the use of Kinect $v 2$ for exercise monitoring, ACM, Proc. of the 2nd International Workshop on Movement and Computing (MOCO '15), pP.112-119, 2015. 2015.

[6] Avola, D, Cinque, L, Levialdi, S, Petracca, A, Placidi, G, Spezialetti, M: Markerless Hand Gesture Interface Based on LEAP Motion Controller Proc. of the 20th International Conference on Distributed Multimedia Systems: Research papers on distributed multimedia systems, distance education technologies and visual languages and computing, pp.27-29, 2014.

[7] Microsoft: Kinect for Windows SDK C++ Reference, https://msdn.microsoft.com/en-us/library/dn791993.aspx (2017/06/08 access)

[8] Nitta, Y.: NtKinect - C++ Class Library for Kinect V2, the 172-th conference SIG Human-Computer Interaction, IPSJ, 2017.

[9] Nitta, Y.: NtKinect - Kinect V2 C++ Programming with OpenCV on Windows10, http://nw.tsuda.ac.jp/lec/kinect2/index-en.html (2017/06/08 access).

[10] Nitta, Y.: NtKinect - How to recognize human skeleton with Kinect V2, http://nw.tsuda.ac.jp/lec/kinect2/KinectV2_ skeleton/index-en.html (2017/06/08 access)

[11] Nitta, Y.: NtKinect - How to recognize human face with Kinect V2 in ColorSpace coordinate system, http://nw.tsuda.ac.jp/lec/kinect2/KinectV2_ face/index-en.html (2017/06/08 access).

[12] Tsuchiya, M., Itoh, T., Nitta, Y.: Interactive Light Painting System Using Human Recognition, NICOGRAPH 2016, P-4, The Society for Art and Science, 2016.

[13] Tsuchiya, M. Itoh, T. Nitta, Y: An Interactive System for Light-Art-Like Representation of Human Silhouettes, WISS 2016, P-213, JSSST, 2016.

[14] Tsuchiya, M., Itoh, T., Nitta, Y.: An Interactive System for Light-Art-Like Representation of Human Silhouettes, Interaction 2017, IPSJ, 2017.

[15] Tokunaga, S. SAIKI, S. Matsumoto, S. Nakamura, M: A Classification Method of Remote Monitoring Service for Elderly Person, Vol. 113, No.469, p.169-174, 2014.

[16] Japan Post: Watching Service of Post Office, http://www.post.japanpost.jp/life/mimamori/index.html (2017/08/29 access) (in Japanese).

[17] Ramrock: Care Support System "Ramrock System”, http://www.ramrock.co.jp/ (2017/08/29 access) (in Japanese).
[18] Zojirushi: MIMAMORI Hot Line, http://www.mimamori.net/ (2017/08/29 access) (in Japanese).

[19] Bradford, D. Freyne, J. Karunanithi, M.: Sensors on My Bed: The Ups and Downs of In-Home Monitoring, vol.7910, Lecture Notes in Computer ScienceSpringer Berlin Heidelberg 2013.

[20] Philips: Medical Alert Service, http://www.lifeline.philips.com/ (2017/08/29 access).

[21] Fujitsu : "The deciding factor is IoT and sound", Elderly monitoring service to the next step http://www.fujitsu.com/jp/innovation/digital/life/dl-contents/ 2017/topics-05/ (2017/06/08 access) (in Japanese).

[22] OpenCV: OpenCV library http://opencv.org/ (2017/06/08 access)

[23] Microsft: Kinect for Windows SDK 2.0, Programming Guide, Body tracking, https://msdn.microsoft.com/en-us/library/dn799273.aspx (2017/09/03 access).

[24] Cabinet Office, Government of Japan: Disaster Management in Japan, http://www.bousai.go.jp/1info/pdf/saigaipanf_e.pdf (2017/09/02 access).

[25] Wikipedia: 2014 Mount Ontake eruption https://en.wikipedia.org/wiki/2014_Mount_Ontake_ eruption (2017/09/02 access).

[26] Murayama, Y., Sasaki, J. and Nishioka, D.: Experiences in Emergency Response at the Great East Japan Earthquake and Tsunami, HICSS-49, P.146-151, 2016.

[27] Van de Walle, B., Turoff, M. and Hiltz, S.R. eds: Information systems for emergency management, M.E. Sharpe, 2009.

[28] Saito, Y., Fujihara, Y. and Murayama, Y.: A Study of Reconstruction Watcher in Disaster Area, Proc. ACM CHI2012, pp.811-814, 2012.

[29] Wikipedia: LPWAN, https://en.wikipedia.org/wiki/LPWAN (2017/09/02 access).

[30] SIGFOX : Radio Technology Keypoints, https://www.sigfox.com/en/sigfox-iot-radio-technology (2017/06/08 access).

[31] LoRa Alliance: LoRa Alliance Technology, https://www.lora-alliance.org/what-is-lora （2017/09/02 access). 